\title{
Rhetorik des Hellenismus: Von Theophrast bis Philodem
}

Beer, Beate

Posted at the Zurich Open Repository and Archive, University of Zurich

ZORA URL: https://doi.org/10.5167/uzh-165462

Book Section

Published Version

Originally published at:

Beer, Beate (2019). Rhetorik des Hellenismus: Von Theophrast bis Philodem. In: Erler, Michael; Tornau, Christian. Handbuch Antike Rhetorik. Berlin/Boston: De Gruyter, 361-382. 


\title{
13 Rhetorik des Hellenismus: Von Theophrast bis Philodem
}

\begin{abstract}
A vital debate about the scientific status of rhetoric arose out of the competition between philosophy and rhetoric as educational subjects. This debate later on caused conflicts between philosophical schools and their different rhetorical doctrines. It stimulated rhetorical theorization in Hellenistic times. Our knowledge though is founded mostly on interpretive testimonies rather than on direct transmission. Theophrastus and Hermagoras were preeminent in the reception of their rhetorical theories. Both can be considered as arguing in favor of the scientific status of rhetoric. Theophrastus seems to have focused on style and to have formulated his rhetoric by developing Aristotle's differentiation of three kinds of speech, correlating them to three kinds of style. Hermagoras sought to define rhetoric through its content and focused on the forensic kind of speech. He was honoured with the title of the 'inventor of rhetoric'. But it is Theophrastus who seems to have hit the zeitgeist by theorizing on styles of literary prose. In fact, our two main direct sources, the anonymous treatise De elocutione and the fragments of Philodemus' Rhetorics, both within their pro-rhetorical position concentrate on questions about style by fusing poetical and rhetorical considerations. Furthermore, Philodemus in his poetics refers to ideas and terminology on style that correspond closely to those in De elocutione.
\end{abstract}

Stichwörter: Theoriebildung, Stilistik, Stasis-Lehre, Forensik, sophistische Rhetorik, Epikureismus, Dichtung, Brief, alexandrinische Philologie, Kunstprosa

Keywords: theorization, stylistics, stasis theory, forensic science, sophistic rhetoric, Epicureanism, poetry, letter, Alexandrine philology, literary prose

Gliederung: 1 Die Debatte um die Wissenschaftlichkeit von Rhetorik -2 Rhetorische Theorie bei Theophrast - 3 Rhetorische Theorie bei Hermagoras - 4 Rhetorische Theorie bei Philodem - 5 Von

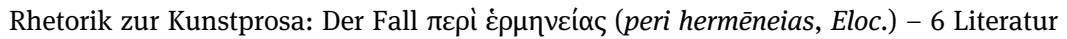

\section{Die Debatte um die Wissenschaftlichkeit von Rhetorik}

Der zeitliche Bogen hellenistischer Rhetorik umspannt die Reflexion Theophrasts von Eresos (371-287) bis Philodems von Gadara (110-40/35). Zwischen diesen peripatetischen und epikureischen Angelpunkten stehen zahlreiche weitere Namen rhetorischer Theoretiker aller vier großen hellenistischen Schulen, die in ihrer Fülle von der Bedeutung rhetorischer Theoriebildung im Hellenismus zeugen, als mit 
Aristoteles' Rhetorica die Schrift, die kanonische Wirkungskraft hätte entfalten können, unbeachtet blieb. So sind aufgrund von Testimonien für zahlreiche Namen neben Theophrast und Philodem - darunter Demetrios von Phaleron (350-280), Chrysippos von Soloi (281/76-208/4), Kritolaos von Phaselis (3. Jh.), Hegesias von Magnesia (3. Jh.), Ariston von Keos (3. Jh.), Hermagoras von Temnos (2. Jh.), Panaitios von Rhodos (180-110), Metrodoros von Stratonikeia (170/65-110), Charmadas (168/7-102/91) sowie Theodoros von Gadara und Apollodoros von Pergamon (beide 1. Jh.) - Schriften zur rhetorischen Theorie belegt. ${ }^{1}$ Dieser philosophischen wie personellen Breite steht eine schmale Überlieferungslage gegenüber. Wir wollen zunächst anhand punktueller Beobachtungen ihr Spektrum abstecken und die Schwierigkeiten bei der Zuschreibung von Aussagen und Haltungen zu den einzelnen Persönlichkeiten illustrieren.

Von Philodems Rhetorica sind zwar umfangreiche Fragmente auf den beim Vesuv-Ausbruch des Jahres 79 n. Chr. verkohlten und in der herkulanensischen Villa dei Papiri gefundenen Papyrus-Rollen erhalten. Allein schon aufgrund ihres fragmentarischen Überlieferungszustandes ist der Inhalt der Schrift aber sehr schwierig fassbar. Überliefert haben wir aus hellenistischer Zeit auch eine Schrift mit dem

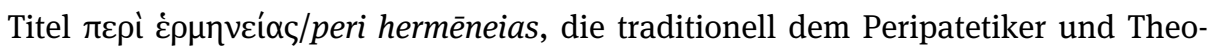
phrast-Schüler Demetrios von Phaleron zugeschrieben wird, letztlich jedoch kaum auf diesen zurückgehen dürfte. ${ }^{2}$ Von in ihrer Produktivität und Nachwirkung so herausragenden Theoretikern wie Theophrast (Diogenes Laertios verzeichnet für Theophrast immerhin rund 20 Titel rhetorischer Thematik) und Hermagoras ist dagegen außer Theophrasts Schrift Characteres gar kein direktes Zeugnis erhalten. Beider Beitrag zur rhetorischen Reflexion muss aufgrund späterer Quellen (insbesondere Cicero, De inventione, Quintilian, Institutio oratoria, und Augustin, De rhetorica) erschlossen werden. Das Bemühen um eine Rekonstruktion gerade der Schriften Theophrasts geht so weit, dass wiederholt Theophrast als Quelle angenommen wird, schon wenn Cicero sich zu einem Thema äußert, zu dem ein Titel von Theophrast überliefert ist. So betrachtet Fortenbaugh 1985, 279 Ciceros Äußerungen in de orat. 3,216 zum Rückschluss auf die Emotionen des Redners aufgrund von Zeichen wie Miene, Stimme und Gestik als Rezeption von Theophrasts Schrift über den Vor-

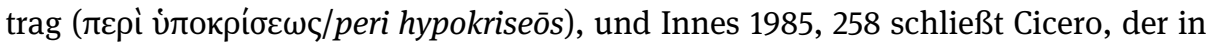
orat. 79 als vierte Redetugend nach Theophrast den angenehmen und reichen Schmuck nennt (ornatum illud suave et affluens) an das Theophrast-Testimonium in

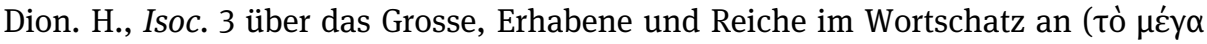

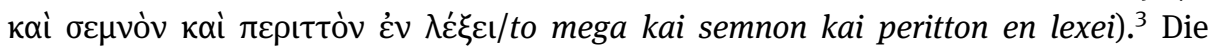

1 Quint. inst. 2,17,15 nennt neben Kritolaos als weitere gegen Rhetorik polemisierende Philosophen Athenodoros von Rhodos und Agnon. Beide werden nur an dieser Stelle genannt.

2 Eine Übersicht über Datierungs- und Zuschreibungsversuche bietet Chiron 2001, 17-32.

3 Fortenbaugh 2005, 59-61 versteht affluens in orat. 79 anders als Innes 1985 als freie Anfügung Ciceros. 
Lehre des Stoikers Panaitios von Rhodos lässt eine Öffnung gegenüber den akademischen und peripatetischen Schulen erkennen, die sich nicht zuletzt aufgrund des römischen Umfelds, in dem Panaitios wirkte, vollzogen haben dürfte. Kennedy 1994, 92 vermutet einen Einfluss von Panaitios, wenn Cicero in off. 1,136 f. ausführt, dass der Redner zwar einen Gegner streng und zornig tadeln könne, sein Zorn dabei aber nur ein vordergründiger und nicht ein empfundener sein solle. Cicero selbst verweist in off. 2,51 auf Panaitios als Quelle für die Haltung, dass es eher die Aufgabe des Redners ist, vor Gericht jemanden zu verteidigen, auch wenn seine Schuld nicht ausgeschlossen werden kann, als jemanden anzuklagen, der möglicherweise unschuldig ist. Kennedy 1994, 93 zieht Cicero auch für Rückschlüsse auf die akademische rhetorische Reflexion in ihren Vertretern Charmadas und Metrodoros von Stratonikeia heran. Denn aufgrund von de orat. 2,360 und Plin. nat. 7,89 lässt sich die vierte Aufgabe des Redners, die memoria bzw. $\mu \nu \eta \dot{\mu \eta} / m n \bar{e} m \bar{e}$, auf Charmadas und Metrodoros zurückführen. ${ }^{4}$ In der akademischen Skepsis hatte die Rhetorik laut Cic. ac. 2,7 ferner als Mittel der Argumentation für und wider eine Auffassung (in utramque partem disputare) eine zentrale Rolle. Von dem hohen Ansehen akademischer Rhetorik, die aber instrumentellen Charakter behielt, zeugt der Umstand, dass Cicero sich für seine rhetorische Ausbildung dem Akademiker Philon von Larisa anschloss. Quintilian (inst. 3,1,17 f.) nennt die beiden Redner Theodoros von Gadara und Apollodoros von Pergamon, die beide als Verfasser rhetorischer Lehrbücher bekannt sind, als Anführer einer Auseinandersetzung über Rhetorik, wie sie sonst nur zwischen verschiedenen Philosophenschulen geführt würde.

Der Grund für diese Fülle von hellenistischen Philosophen und Schriftstellern, die über Rhetorik debattiert haben, ist nach Liebersohn 2010 in der Konkurrenzsituation zu sehen, in der Rhetorik als neueres Studienfach der älteren Philosophie gegenüber stand. Die Situation ist im Hellenismus zwar nicht neu, von ihr zeugt schon Platon mit seinem Dialog Gorgias. Doch erkennt Liebersohn 2010, $24 \mathrm{f}$. eine Verschärfung, als sich nach den Eroberungen Alexanders des Großen in der Hellenisierung neuer Gebiete Rhetorik als geeigneter erwies, den Anschluss an die griechische Oberschicht zu gewährleisten. Liebersohns These wird gestützt durch die weitere Entwicklung bis zum Attizismus der Zweiten Sophistik, wo sich wiederum die Sprache als manifester Ausdruck der Zugehörigkeit zur Oberschicht erweist. So folgt auf Platons Anfänge die hellenistische Fortsetzung der Rhetorik-Kritik, deren Rekonstruktion Liebersohn aufgrund von späteren Quellen wie Cicero, De oratore und Quintilian unternimmt. Demnach scheint auf philosophischer Seite zunächst Kritolaos federführend gewesen zu sein, auch wenn dies letztlich schwierig zu bestimmen ist, da Quintilian die hellenistischen Wortführer der Argumente gegen die

$4 \mathrm{Zu}$ den fünf Aufgaben des Redners von der Bestimmung des Themas bis zum Vortrag vgl. Cic. de orat. 1,142 . 
Wissenschaftlichkeit von Rhetorik oftmals zusammenfassend behandelt. ${ }^{5}$ Liebersohn schreibt Kritolaos die Argumente zu, wonach 1. Rhetorik auf Erfahrung ( $\tau \rho \wedge \dot{\text { / }}$ / tribē) und nicht auf Unterricht beruhe; ${ }^{6}$ 2. Rhetorik kein thematisch abgrenzbares Gebiet umschließe und daher eine ars circumcurrens sei; ${ }^{7} 3$. Rhetorik, wenn schon

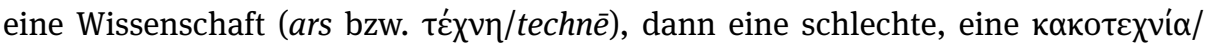
kakotechnia sei, weil sie mit falschen Meinungen arbeite und der Gesellschaft schade ${ }^{8}$ 4. Rhetorik mit der Überzeugung kein ihr spezifisch zuweisbares Ziel verfolge, mithin überhaupt eine ó $\tau \varepsilon \chi v i ́ \alpha / a t e c h n i a$ sei. ${ }^{9}$ Anders hat sich die Stoa mit der wachsenden kulturellen Bedeutung von Rhetorik auseinandergesetzt und ihre Beherrschung zu den Attributen des stoischen Weisen gerechnet. Liebersohn 2010, $32 \mathrm{f}$. entnimmt dies wiederum Testimonien wie Cic. de orat. 3,65 sowie Quint. inst. 2,17,2. Cic. fin. 4,7 erwähnt ferner Rhetoriken von Kleanthes und Chrysipp. ${ }^{10}$ Die Stoiker setzen also eine sittlich gute, nicht der Manipulation dienende Rhetorik an. Damit schließen sie die Redelehrer aus der Diskussion aus und bestimmen die Rhetorik nach Quint. inst. 2,15,34 als bene dicendi scientia (,Wissenschaft vom guten Reden“), wobei sich in Quintilians Erläuterung „gut“ sowohl auf das Wesen des Redners als vir bonus als auch auf die Rede selbst mit ihren spezifischen Tugenden bezieht. ${ }^{11}$ Diese Definition, durch die Rhetorik zwar inhaltlich an die philosophische Unterweisung gebunden wird, aber innerhalb von dieser Unterricht in Stilistik erfordert, hat die Gegenposition der contra argumentierenden Akademiker angeregt. Liebersohn 2010, 128 sieht Charmadas an vorderster Front dieser philosophie-internen Debatte, die sich auch um den Nutzen einer so definierten Rhetorik dreht. Ferner kann Philodems epikureisches Konzept einer sophistischen Rhetorik, welche nur der Philosoph beherrscht und Probleme wie den Redeaufbau und die Wortwahl umfasst, als Auseinandersetzung mit der stoischen Rhetorik-Definition und ihrer Abwendung von der Überzeugung als traditionellem Ziel der Rhetorik ${ }^{12}$ hin zur guten Rede verstanden werden.

Aus dieser Vielzahl hellenistischer Rhetoriker sollen im Folgenden die beiden mit der größten belegten Nachwirkung, der Peripatetiker Theophrast und Hermago-

5 Ausdrücklich nennt Quint. inst. 2 dagegen immer wieder Platons Gorgias, der von den RhetorikGegnern verschiedener Schulen als ergiebige Quelle für ihre Argumente genutzt worden sein dürfte. Diesen Eindruck vermittelt auch Aug. rhet.

6 Quint. inst. 2,15,23.

7 Quint. inst. 2,21,7-11.

8 Quint. inst. 2,15,2 und 23 sowie 2,20,2.

9 Quint. inst. 2,20,2.

10 Die Fragmente von Chrysipps Rhetorik gibt SVF 2,298a wieder.

11 Quintilian übernimmt diese stoische Definition von Rhetorik für sich selbst und wiederholt sie in inst. 2,15,38.

12 Quint. inst. 2,15,3: est igitur frequentissimus finis ,rhetoricen esse vim persuadendi‘. („Die Definition, dass die Rhetorik die ,Fähigkeit zur Überzeugung' sei, ist also die häufigste“); vgl. 2,15,14 zu Hermagoras; ebenso Aug. rhet. 2: ergo quasi consensu omnium finis est oratoris officii persuadere („Folglich ist in Übereinstimmung beinahe aller das Ziel der Redneraufgabe das Überzeugen“). 
ras, vorgestellt werden. Ihnen wird als Dritter mit Philodem derjenige zur Seite gestellt, der in der späteren Antike völlig unbeachtet blieb, dem der Zufall der Überlieferung aber am günstigsten war. Allein der Umstand, dass er als Epikureer eine Schrift zur Rhetorik verfasst hat, verdeutlicht, wie breit rhetorische Theorie diskutiert wurde und wie sehr eine Stellungnahme zum Thema von einem hellenistischen Philosophen erwartet wurde. ${ }^{13}$ Theophrasts und Philodems spezifisch hellenistischer Zugang zu Rhetorik, wenn es darum geht, ihre Wissenschaftlichkeit und ihren Stellenwert in der Jugenderziehung zu erörtern, soll durch den abschließenden Ver-

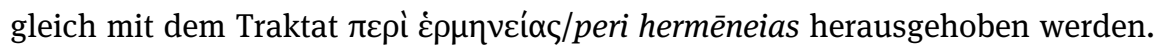

\section{Rhetorische Theorie bei Theophrast}

Theophrasts wichtigster Beitrag zur rhetorischen Theoriebildung wird allgemein in der Unterscheidung der drei Redestile - erhaben, mittel und einfach - einerseits und der Bestimmung der vier Redetugenden - 1. sprachliche Korrektheit, 2. Klarheit, 3. Angemessenheit und 4. Schmuck - andererseits gesehen. Die Anbindung solch stilistischer Klassifikation an Theophrast scheint allerdings eher auf einer antiken Tradition zu gründen, als dass sie sich eindeutig belegen ließe. Die Unterscheidung dreier Stile ergibt sich aus Dionysios von Halikarnassos, Dem. 3, der den dritten als gemischten beschreibt und unter Rekurs auf Theophrast auf Thrasymachos von Chalkedon zurückführt. Indem Theophrast den drei aristotelischen Redegattungen

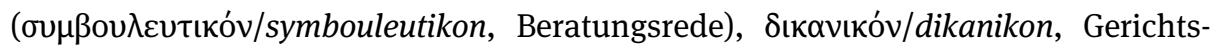

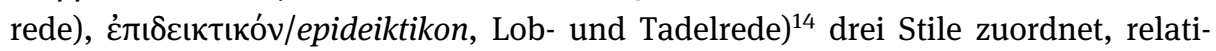
viert er Aristoteles' Konzentration auf den Inhalt der Rede. Zeugnis dafür, dass der Stil im Peripatos allgemein und insbesondere von Theophrast erörtert wurde, gibt Cic. de orat. 1,43 und 55. Später, in 3,49-53, führt Cicero die einzelnen Tugenden auf als 1. „Regeln der lateinischen Sprache“ (praecepta Latine loquendi = latinitas

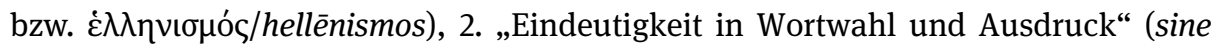
ambiguo verbo et sermone = perspicuitas bzw. $\sigma \alpha \varphi \eta ́ v \varepsilon ı /$ saphēneia), 3. „was ich ,angemessen, passend" nennen möchte“ (quod ego aptum et congruens nominem = ap-

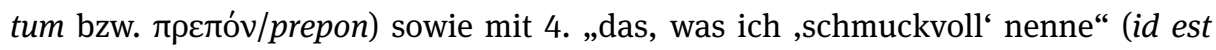

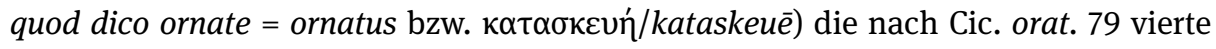
theophrastische Redetugend. Mit der Bestimmung der Redetugenden behandelt Theophrast die elocutio bzw. $\lambda \varepsilon^{\prime} \xi ı \varsigma / l e x i s$ (sprachliche Gestaltung) als dritte Aufgabe des Redners. Theophrast dürfte die Begrifflichkeit aufgenommen haben, in der sich

13 Arrighetti 2000, 18 f. skizziert die gängige ablehnende Haltung des Kepos seit Epikur gegenüber kulturellen Studien.

14 Aristot. Rh. 1,3, $1358 \mathrm{~b}$. 
Aristoteles in der Rhetorik über stilistische Tugenden äußert. ${ }^{15}$ Dionysios von Halikarnassos bietet in Isoc. 3 einen Beleg dafür, dass Theophrast den Redeschmuck

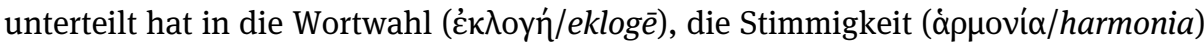
unter den gewählten Wörtern und die sich aus der Anordnung der Wörter ergeben-

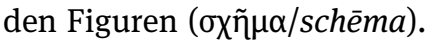

Auch abgesehen von diesem Schematismus, der eine Technisierung der Rhetorik nahelegt, wird Theophrasts weitere Rezeption seines Lehrers Aristoteles fassbar, der wiederum nach Cicero (Tusc. 1,7) als erster Philosoph die Notwendigkeit erkannte, die Rhetorik in das philosophische Curriculum aufzunehmen. In der Forschungsliteratur gehen die Meinungen über die Bewertung von Theophrasts eigenem Beitrag jedoch auseinander. Anders als Kennedy 1994 ist Grube 1952 generell zurückhaltend. Seiner Ansicht nach geht Theophrast einzig in dem Zeugnis Dem. Eloc. 222 - wo ihm die Aussage zugeschrieben wird, dass eine Rede an Überzeugungskraft gewinne, wenn dem Hörer die Argumentation nicht allzu detailliert ausformuliert werde, so dass ihm Raum bleibe, um eigene Schlüsse zu ziehen - über Aristoteles hinaus. Doch kann sich Theophrast auch für diese Aussage an Aristoteles $\left(R h .1,11,1371 \mathrm{~b}\right.$ und 2,23, 1400b) orientiert haben. ${ }^{16}$ Über Einzelbeobachtungen dieser Art hinaus wollen wir unser Augenmerk auf die grundsätzliche Bewertung des Stils bei Aristoteles und Theophrast richten. In Rh. 3,1, 1404a beschreibt Aristoteles den Stil als ein Element, das die Rhetorik von der Dichtung übernommen hat. Er spricht ihm mithin eher periphere Bedeutung für die Rhetorik zu, die, so Aristoteles, allein aufgrund der Präsentation von Fakten überzeugen sollte und nur wegen

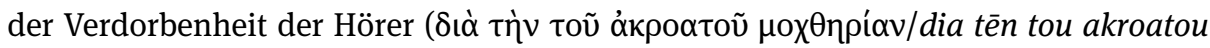
mochthèrian) auch auf die emotionale Wirkung des Stils angewiesen sei. Wenn Theophrast den Stil dagegen ins Zentrum seiner rhetorischen Reflexion rückt, etabliert er gerade eine Nähe von Rhetorik und Poesie bzw. prosaischer und dichterischer Sprache. Ein ähnliches Bild ergibt sich bei Dion. H. Comp. 16,15 zu Theophrasts Einteilung der Wörter in schöne und erhabene einerseits und mittelmäßige und niedrige, mittels derer ebensowenig gute Dichtung wie gute Rede geschaffen werden könne, andererseits. Auch Quint. inst. 10,1,27 legt nahe, dass Theophrast Gemeinsamkeiten von Rhetorik und Dichtung in den Blick genommen hat:

Plurimum dicit oratori conferre Theophrastus lectionem poetarum multique eius iudicium sequuntur.

Theophrast sagt, dass die Lektüre von Dichtern für den Redner sehr gewinnbringend sei, und viele folgen seinem Urteil.

15 Fuhrmann 1995, 114-145 gibt einen Überblick über die Terminologie, die Grube 1952, 180 für

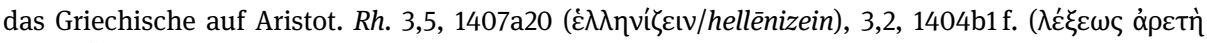

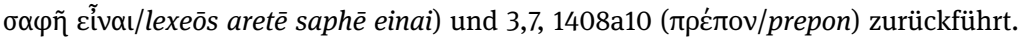

16 So auch Kennedy 1994, 85 zur Stelle: „reminiscent of Aristotle“. 
Offenbar galt Theophrast für Cicero, Quintilian und Dionysios von Halikarnassos als Vorbild in der Erörterung des Redestils. Dabei ist nicht auszuschließen, dass diese Vorbildfunktion durch die Etymologie zu Theophrasts Namen motiviert ist. So führt Diogenes Laertios $(5,38)$ aus, dass Theophrast ursprünglich Tyrtamos geheißen und wegen seiner angenehmen Ausdrucksweise seinen späteren, sprechenden Namen von Aristoteles erhalten habe. ${ }^{17}$ Theophrast weist damit den Weg zur Entwicklung einer Kunstprosa und der Instrumente ihrer Kritik, wie sie nur eine Generation später in Alexandria als Mittel zur Dichtungskritik erarbeitet wurden. ${ }^{18}$ Rhetorik im Hellenismus ist in der Regel auch Stilkritik und als solche von der Stilkritik zeitgenössischer Dichtungstheorie schwierig zu trennen. Nicht nur Philodem hat mit Rhetorica und De poematis beiden Formen künstlerischer Sprachgestaltung Traktate gewidmet. Schon Aristoteles hat neben der ars rhetorica eine poetica verfasst.

Auf eine Bestimmung von Kunstprosa durch Theophrast weist auch der Um-

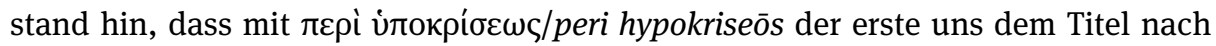
bekannte Traktat, der dem Redevortrag (actio) als der fünften und finalen Aufgabe des Redners gewidmet ist, von ihm stammt. Der Vortrag, der wie der Stil nach Aristoteles ebenfalls auf die Hörerlenkung ausgerichtet ist, kann mit Fortenbaugh 2005, 52 als zweites Thema gelten, das Theophrast gegenüber der Behandlung bei Aristoteles ausführlicher ausgearbeitet hat. Cicero (de orat. 3,221) stützt sich bei der Behandlung des Blickkontaktes des Redners zu den Hörern ausdrücklich auf Theophrast. ${ }^{19}$ Auch hier kann vermutet werden, dass Theophrast Aristoteles' Lehre weiter entwickelt, indem er zusammenfasst, was Aristoteles punktuell in $R h .3,1,1403 \mathrm{~b}$ (Stimme) und in $R h .3,12,1413 \mathrm{~b}$ (Stilelemente, die beim Redner eine bestimmte Gestik generieren) und in der Poetik ${ }^{20}$ zum Vortrag allgemein behandelt. Gleichzeitig bekräftigt Theophrast durch die Behandlung des Vortrags, der ja traditionell zu den Aufgaben von Dichtern und später von Rhapsoden und Schauspielern gehört (vgl. Aristot. Rh. 3,1, 1403b und Dem. Eloc. 195), die in der Behandlung des Stils festzustellende Annäherung von Rhetorik an die Literaturkritik. Fortenbaugh 2003, 271 schließt aus den Testimonien, dass Theophrast den Vortrag umfassend in Bezug auf den Redner, den Musiker, den Schauspieler und den Sänger behandelt habe. Dass die Erörterung des Vortrags die Auseinandersetzung mit dem Stil weiterführen kann, zeigen Dem. Eloc. 193 und 271 sowie Philod. Rh. 4,201, col. 19a,16-26, die den Vortrag durch den Stil und das Umfeld des Redners bestimmt sein lassen.

17 Eine ähnliche Etymologisierung des Namens ist zu Pythagoras bekannt; vgl. Diog. L. 8,21 unter Referenz auf Aristipp. Philodem in epigr. 10 (Sider) spielt diese Praxis an seinem eigenen Namen durch.

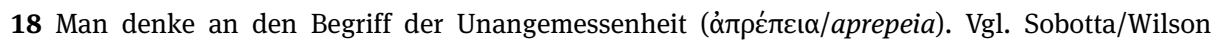
2000, 839.

$19 \mathrm{Zu}$ den Schwierigkeiten bei der Deutung der Stelle vgl. Fortenbaugh 2003, 259.

20 Aristot. Poet. 6, 1450a und 26, 1462a (Inszenierung); 17, 1455a (Affekte des Schauspielers); 20, 1456b; 25, 1461a; 26, 1462a (Interpretation durch die Intonation des Schauspielers). 
Fortenbaugh 1994 rechnet mit den unterhaltsamen Characteres auch die bekannteste und am besten erhaltene Schrift Theophrasts zur rhetorischen Theorie. ${ }^{21}$ Er führt einleuchtend aus, dass die von Theophrast beschriebenen Charakterzüge späteren Rednern als Quelle für ihre eigenen Charakterdarstellungen gedient haben könnten, und belegt seine Vermutung durch Beispiele praktischer Rhetorik. Die Characteres können demnach als Rhetorik-Handbuch, das der Redner als Inspi-

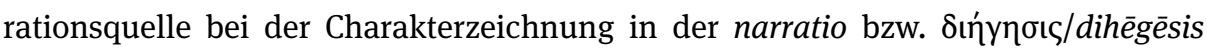
(Darstellung des Sachverhalts) heranzog, gelesen werden. Auch hierin ist eine Literarisierung der Rede zu sehen. Eine Rede, in der die Charakterzeichnung so großes Gewicht erhält, verfolgt nicht nur eine wahrheitsgetreue, an Objektivität orientierte Zusammenstellung von Fakten sondern auch eine Fiktionalisierung von Personen und Handlung ${ }^{22}$ im Dienste des Wahrscheinlichen, das Aristoteles (Poet. 6, 1450ab und 9, 1451a-b) als Spezifikum fiktionaler Literatur bestimmt. Auch der Dichter muss seinen Gegenstand für seine Adressaten überzeugend darstellen. Aristoteles (Poet. 15, 1454a) und Horaz (ars 119-130 und 152-178) halten fest, dass dies über eine kohärente Figurendarstellung erreicht werden kann. In dieser gemeinsamen Perspektive von Dichtung und Rhetorik auf das Wahrscheinliche im Unterschied zum Wahren ist auch der Ausgangspunkt von Platons Rhetorik- und Dichtungsskepsis zu verorten. ${ }^{23}$ Theophrast führt in seiner Stilistik konsequenterweise beide, Rhetorik und Dichtung, zusammen.

\section{Rhetorische Theorie bei Hermagoras}

Wie Theophrast gilt auch Hermagoras von Temnos als Schüler des Peripatos. Mit Theophrast verbindet ihn neben der starken Nachwirkung auch das Bestreben nach einer Systematisierung von Rhetorik, als deren Ziel die Etablierung von Rhetorik als wissenschaftlicher Disziplin zu sehen ist. Dem rhetorischen Ziel der Überzeugung schaltet Hermagoras die Beherrschung der Theorie als Ziel vor. Anders als Theophrast scheint Hermagoras sich aber nicht auf die Redneraufgaben der elocutio bzw.

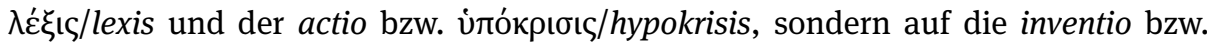

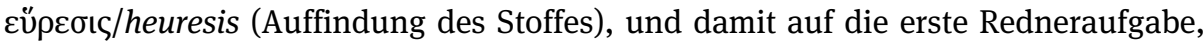
konzentriert zu haben. Die Testimonien vermitteln nachgerade das polemisch gefärbte Bild eines dem Redeschmuck abgeneigten Rhetorikers, der selbst einen sehr nüchternen Stil pflegte. ${ }^{24}$ Dieses Bild erhält in seiner Repetition in den Testimonien

21 Woerther 2012, vii folgt Fortenbaugh 1994.

22 Vgl. van Mal-Maeder 2007, 41-64 zur Prosopopoiie in den Deklamationsübungen der römischen Rhetorik und zur Erzählperspektive im Roman.

23 Bes. Plat. Gorg. bzw. Rp. 10,595a-597e unter dem Aspekt der Mimesis. Motivation und Ausmaß der Dichtungskritik bei Platon relativiert Erler 2007, 486-497.

24 Allen voran polemisiert Cicero, der selbst beispielsweise in Tusc. 1,7 einen schmuckvollen Stil favorisiert, in Brut. 263 und 271 sowie inv. 1,8: verum oratori minime est de arte loqui, quod hic fecit, 
klischeehafte Züge. Den Eindruck der Geringschätzung auch von Hermagoras' theoriebildender Leistung weckt Plutarch, der in Pomp. 42,5 eine Szene überliefert, wonach Pompeius in Rhodos vor Poseidonios gegen Hermagoras' Stasis-Lehre deklamierte.

Hermagoras belegt also die Technikalität von Rhetorik im Gegensatz zu Theophrast weniger, indem er sie an eine andere, anerkannte Disziplin wie der Dichtung anlehnt, sondern indem er vielmehr ein inhaltliches Spezifikum sucht. Daher ist es auch Hermagoras und nicht Theophrast, dem ein eigenständiger Definitionsversuch von Rhetorik zugeschrieben wird, dessen Nachwirkung in den von Woerther 2012 zusammengestellten Testimonien bis ins 5. Jh. n. Chr. zu verfolgen ist. Rhetorik, so Hermagoras gemäß Augustin, rhet. 3, befasst sich mit der Rede zu öffentlichen Angelegenheiten:

dicit enim esse oratoris officium persuadere, quatenus rerum et personarum condicio patitur, dumtaxat in civilibus quaestionibus.

Er sagt nämlich, dass die Aufgabe des Redners sei zu überzeugen, soweit es die äußeren und Personen betreffenden Umstände zulassen, zumindest in öffentlichen Fragen.

Diesem auf einen spezifisch rhetorischen Gegenstand ausgerichteten Zugang war in der Nachwirkung insofern mehr Erfolg beschieden, als Hermagoras in späteren Quellen trotz des grundsätzlich polemischen Tenors allgemein als der Erfinder der Rhetorik inszeniert wird. ${ }^{25}$ Rhetorik gerät bei Hermagoras, so werden wir sehen, allerdings zu einer forensischen Disziplin.

Hermagoras gilt, wenn nicht als Begründer der Stasis-Lehre (so Woerther 2012, xxi, die selbst die Anfänge der Stasis-Lehre bei Aristoteles sieht), so doch als ihr Theoretiker, indem er die Praxis begrifflich definiert hat. Ihr Schwerpunkt liegt auf der Gerichtsrede, der nach Aristoteles ersten rhetorischen Gattung (genus iudiciale

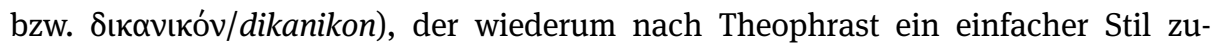
kommt. Ein Verständnis der Stasis-Lehre ist nur schwer zu erlangen, weil sie indirekt über Testimonien erschlossen werden muss. Selbst der Zugriff auf Hermagoras' Terminologie ist aufgrund der fast ausschließlich lateinischen Testimonien erschwert.

Die Stasis-Lehre vermittelt dem Redner ein Instrumentarium zur Bestimmung des Themas in einer Kontroverse (controversia). Nach Cicero (inv. 1,8) hat Hermagoras den Redestoff in quaestio und causa unterteilt. Augustin (rhet. 7) bietet dazu die

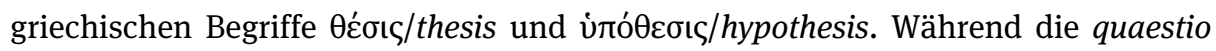

multo maximum ex arte dicere, quod eum minime potuisse omnes videmus („Aber es gehört sich für einen Redner keineswegs, über die Kunst zu sprechen, was dieser [sc. Hermagoras] gemacht hat, vielmehr [gehört es sich] aus der Kunst heraus zu sprechen; dass er dies überhaupt nicht beherrschte, sehen wir alle“). Die Kritik wird übernommen von Tac. dial. 19,3: aridissimis Hermagorae [...] libris (,in den saft- und kraftlosen Büchern von Hermagoras“).

25 So Woerther 2012, lxxi über Hermagoras bes. in Cicero, De inventione. Ciceros Haltung referiert Quint. inst. 3,5,14. 
Kontroversen bezeichnet, die unabhängig von konkreten Personen und Umständen geführt werden, handelt die causa von Kontroversen, die sich nach Personen und Umständen als Einzelfall bestimmen lassen. Diese erste Unterteilung in quaestio und causa läuft nach Ciceros Darstellung in inv. 1,8 auf eine Unterscheidung von Fragen, wie sie in den Philosophieschulen diskutiert wurden (ob bspw. der sinnlichen Wahrnehmung Wahrheitsgehalt zukommt oder nicht) und Fragen, wie sie vor Gericht debattiert werden (ob beispielsweise die Person x zum bestimmten Zeitpunkt die Person y ermordet hat oder nicht) hinaus. ${ }^{26}$ In de orat. 2,134 f. bestreitet Cicero den Nutzen einer Unterscheidung in quaestio und causa, da jede causa zu ihrer Beurteilung verallgemeinert werden müsse. ${ }^{27}$ Gegen Cicero, der wiederholt insbesondere gegen Hermagoras' Stil polemisiert und vielleicht auch in inv. 1,8 kein objektiver Gewährsmann ist, ist festzuhalten, dass sich jede causa unter Wahrung des juristischen Kontextes als quaestio abstrahieren lässt. Mit der Bestimmung der quaestio begründet Hermagoras also nicht zwingend eine Konkurrenz zwischen Rhetorik und Philosophie. Ihr kann auch eine Unterscheidung innerhalb des genus iudiciale zwischen Legislatur (quaestio) und prozessualer Rechtssprechung (causa) zugrunde gelegt werden. Quintilian (inst. 3,5,12-14) legt Zeugnis ab von der Debatte, die um das Verständnis von Hermagoras' quaestio bereits in der Antike geführt wurde. Die diesbezüglichen Unsicherheiten betreffen auch eine weitere Unterteilung innerhalb der quaestiones in quaestio universalis und quaestio finita und erstrecken sich auf die Frage, ob quaestiones überhaupt Gegenstand des Redners sind oder nicht. Aelius Theon (Prog. p. 120,13-18 Spengel) unternimmt eine Bestimmung der quaestio unter Abgrenzung vom Topos, der zu entnehmen ist, dass die quaestio als Kontroverse formuliert sei und eine Beurteilung erfordere.

Augustin (rhet. 7) und Quintilian (inst. 3,5,17) überliefern uns ferner als Unterteilung innerhalb der causa anhand von sieben Peristasen (circumstantia rerum) die Umstände, die aufgrund der Fragen nach dem quis, quid, quando, ubi, cur, quem ad modum und quibus adminiculis bestimmt werden können. Die Peristasen bündeln sich in der Stasis (status), mit der die Verteidigung ${ }^{28}$ den strittigen Punkt bestimmen und zu ihrer zentralen Aussage finden kann. Sie machen nach Fuhrmann 1995, 99 die These (quaestio) zur Hypothese (causa).

Innerhalb der Stasis sind nach Quint. inst. 3,5,4 zwei Arten zu unterscheiden:

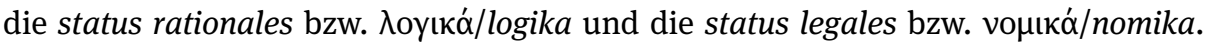
Erstere sind ähnlich den „technischen Beweismitteln“ (probationes artificiales) ${ }^{29}$

26 Mit der quaestio überträgt Hermagoras nach Kasulke 2005, 43 „ethisch-politische[s] Wissen“ aus der Philosophie in die Rhetorik, während im Gegenzug Philosophen wie Philon von Larisa Rhetorikunterricht anboten; vgl. Woerther 2012, $82 \mathrm{f}$.

27 Barwick 1963, 58-60 legt nahe, dass Cicero sich in seiner Kritik auf Vertreter der Akademie und des Peripatos stützt.

28 Für ,Verteidigung، kann im Folgenden auch immer ,Anklage‘ gesetzt werden.

$29 \mathrm{Zu}$ den probationes artificiales und inartificiales vgl. Quint. inst. 5,1,1 unter Referenz auf Aristoteles. 
Streitfragen, die argumentativ zu lösen sind. Gemäß Quint. inst. 3,6,51-62 sind sie unter Referenz auf Hermagoras weiter zu unterscheiden nach coniectura (Tatbestand strittig), nomen (Benennung strittig), qualitas (Bewertung strittig) und translatio (Zuständigkeit des Gerichts strittig): 1. Der status rationalis, der als coniectura bezeichnet wird, stützt sich bei Cic. inv. 1,10 auf den Sachverhalt. Fuhrmann 1995, 104 folgt bei der Erläuterung des status coniecturalis Cic. inv. 2,14: Nach einem Mord steht zur Debatte, ob der Angeklagte tatsächlich der Täter ist oder nicht. 2. Wenn sich ein status rationalis um die Benennung des Falles dreht, gilt er als status nominis. In diesem Fall gibt der Angeklagte die Tat zwar zu, plädiert aber dafür, ihr einen anderen Rechtsverstoß (bspw. Diebstahl vs. Raub) zugrunde zu legen (vgl. Quint. inst. 3,6,41). 3. Nimmt der Verteidiger die Umstände des Falles in den Blick, dann äußert er sich zum status rationalis qualitatis. Im Extremfall kann bei der Diskussion eines Rechtsfalls unter dem Gesichtspunkt des status qualitatis der Täter als Opfer dargestellt werden, wenn Notwehr geltend gemacht wird. 4. Durch den status rationalis translationis lehnt der Verteidiger das Verfahren unter formal-juristischer Argumentation gänzlich ab. So kann etwa dem Kläger das Klagerecht abgesprochen werden. Augustin bietet auch hier teilweise die griechischen Begriffe. Nach Aug.

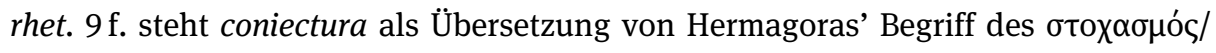
stochasmos und translatio als diejenige von $\mu \varepsilon \tau \alpha \dot{\lambda} \lambda \eta \psi \mathrm{s} /$ metalēpsis.

Die entsprechende Unterteilung der status legales, d.h. der Streitpunkte, die nicht rein argumentativ, sondern aufgrund eines Gesetzestextes zu lösen sind, bietet Consultus Fortunatianus, rhet. 1,22-25: 1. scriptum et voluntas: Hier strebt der Verteidiger eine Debatte um die Frage an, ob ein Gesetzestext wörtlich oder sinngemäß auszulegen sei. 2. leges contrariae: Hier stellt der Verteidiger dem Gesetz, auf das sich die Anklage stützt, ein widersprechendes entgegen, dessen Anwendung er für den debattierten Fall fordert. 3. ambiguitas: Der Verteidiger plädiert für eine andere Interpretation des Gesetzestextes, wobei er sich auf eine mehrdeutige Formulierung desselben stützt. 4. collectio: Der Verteidiger stellt die Behauptung auf, dass der Fall gesetzlich nicht geregelt sei und nennt ein Gesetz, das analog zur Anwendung kommen soll. Fortunatianus, der in die Zeit des 4./5. Jhs. fällt, gibt sich in seiner Bestimmung der Rhetorik in rhet. 1,7 f. ganz der Tradition des Hermagoras verpflichtet, wie sie auch Aug. rhet. 3 vermittelt: Quis finis? - Persuadere, quatenus rerum et personarum condicio patiatur in civilibus quaestionibus (,Was ist das Ziel der Rhetorik? - Zu überzeugen in öffentlichen Fragen, soweit es die Verfassung von Sachverhalten und Personen zulässt“"). Ältere Quellen für die status legales des Hermagoras sind Cicero, inv. 1,17 und Quintilian, inst. 3,6,60-67.

Auffallend ist die wiederkehrende schematische Vierteilung innerhalb der status, die im Dienste einer besseren Memorierbarkeit zu sehen ist, wie Fuhrmann 1995 auch grundsätzlich Hermagoras' Orientierung am Rhetorikschüler betont. Die Hermagoras zugeschriebene Stasis-Lehre trägt die Charakteristik, die für die hellenistische Zeit generell festzustellen ist, wenn es darum geht, Rhetorik umfassend darzustellen und als System zu entwerfen. Die Begriffe bilden eine Pyramide, in der 
sie definiert und weiter ausdifferenziert werden. Der Lehre von Hermagoras folgt auch die anonym überlieferte und in die 80er Jahre des 1 . Jh. v. Chr. datierte Rhetorica ad Herennium. ${ }^{30}$

\section{Rhetorische Theorie bei Philodem}

Anders als Theophrast und Hermagoras hat Philodem zum Beleg der Wissenschaftlichkeit von Rhetorik kein systematisches Handbuch vorgelegt. Die Fragmente seiner Rhetorica legen vielmehr den Schluss nahe, dass Philodem sich unmittelbar mit der philosophischen Debatte auseinandergesetzt und deren Standpunkte referiert und kommentiert hat, um innerhalb derselben zu einer epikureischen Haltung zu gelangen, die zugleich den zeitgenössischen Ansprüchen gerecht wurde, indem sie die wachsende Bedeutung von Rhetorik im Hellenismus berücksichtigte, an der spätestens seit dem 2. Jh. v. Chr. offenbar kein Weg mehr vorbei führte. Der primär epikureische Adressatenkreis der Rhetorica mag - neben dem Vesuvausbruch des Jahres 79 - mit ein Grund für die fehlende antike Nachwirkung des Traktats sein. Philodem steht hier wie auch mit anderen Schriften (beispielsweise De poematis) für eine Öffnung der traditionell dogmatisch an Epikur orientierten Schule, so dass es gerechtfertigt scheint, für die Zeit um $100 \mathrm{v}$. Chr. vom Jungepikureismus als einer neuen Phase innerhalb des Kepos zu sprechen. ${ }^{31}$

Nicht von ungefähr sind es Philodems Rhetorica, die uns Zeugnis geben von der Dynamik innerhalb des Kepos, welche die Impulse hellenistischer Rhetorik aufnimmt. Philodem ist in Rh. 2 (PHerc. 1674), col. 53,7-25 zu entnehmen, dass sich Vertreter der epikureischen Schulen auf Rhodos und Kos in einer Replik (ávtурафи́/antigraphē) gegen eine Aussage des Schuloberhauptes in Athen, Zenons von Sidon gestellt hatten. Dieser hatte erklärt, dass einer bestimmten Form von Rhetorik der Rang einer Wissenschaft zuzuerkennen sei. Philodem schließt sich in der Auseinandersetzung um diese Erklärung seinem Lehrer Zenon $\mathrm{an}^{32}$ und wendet sich gegen die außerathenischen Vertreter, die unter dem Hinweis, dass eine solche Aussage von Epikur nicht überliefert sei, konservativ argumentiert hatten.

Wie weit Philodem in der weiteren Darstellung Zenon folgt, ist aufgrund der fragmentarischen Überlieferung nicht zu bestimmen. Deutlich wird hingegen, dass er für diese besondere Form wissenschaftlicher Rhetorik die Bezeichnung бо

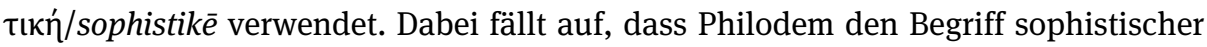
Rhetorik breit diskutiert, während er in den anderen für diesen Beitrag herangezo-

30 Siehe dazu den Beitrag von Thomas Riesenweber in diesem Band, Abschnitt 3.

31 Zur eklektischen Orthodoxie bei Philodem vgl. Erler 1992.

32 PHerc. 1005, col. 14,6-13 ist zu entnehmen, dass Philodem sich als Schüler Zenons von Sidon betrachtet. 
genen Texten nicht verwendet wird. Während in Philod. Rh. 2,214, col. 10,16-18 33 die beiden Gattungen beratender und forensischer Rede genannt werden, weswegen man versucht ist, die sophistische Rhetorik daneben zu stellen und mit der dritten, epideiktischen Gattung gleichzusetzen (so Ferrario 1981, 151 und Arrighetti 2015, 184 f.), scheint sie in Rh. 2 (PHerc. 1674), col. 37,22-31 die beiden anderen Gattungen übergeordnet zu umfassen:

$\tau]$ ò тท่̀ $[\sigma] \mathrm{o}[\varphi \iota \sigma] \tau \iota-$

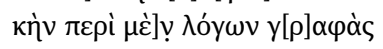

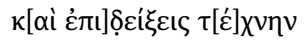

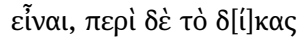

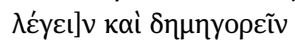

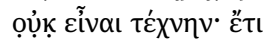

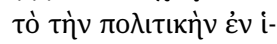

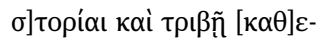

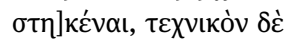

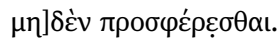

[...], dass sophistische Rhetorik, soweit es um das Schreiben und Vortragen von Reden geht, eine Wissenschaft ist, aber keine Wissenschaft ist, soweit es um Rechtssprechung und Lenkung des Volkes geht; dass außerdem die Rhetorik im öffentlichen Kontext ${ }^{34}$ in Nachforschung und Übung besteht, aber nichts Wissenschaftliches aufweist.

Die sophistische Rhetorik bezeichnet hier als Oberbegriff die von einer thematischen Bestimmung abstrahierte Rhetorik. Diese ist formal ausgerichtet, woraus allein sie ihre Wissenschaftlichkeit bezieht. Anders als bei Hermagoras trägt bei Philodem der Inhalt nichts zur Definition des Faches bei. So sind Rechtssprechung und Lenkung

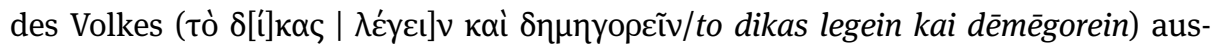
drücklich keine definientia von Rhetorik. Gemäß der Bedeutung des Adjektivs, das von борıбтńs (sophistēs), womit der Könner und Gelehrte als Lehrer von Grammatik und besonders Rhetorik bezeichnet wird, abgeleitet ist, kann sophistische Rhetorik zunächst als Rhetorik der Redelehrer und weiter als ,formale Rhetorik' gedeutet werden, die als Arbeit an der Sprache und in Weiterführung des Sprach- und Literaturunterrichts $\mathrm{zu}$ verstehen ist, der das Curriculum des antiken (Grund-)Schülers fast ausschließlich dominierte. Sie ist bei Philodem insofern die eigentliche Rhetorik, als sie inhaltlich unbestimmt und daher nicht von vornherein im Umfeld eines anderen Gebietes wie Politik oder Forensik angesiedelt ist. Aufgrund ihrer völligen Ausgrenzung inhaltlicher Kriterien kommt sie der epideiktischen mit ihrer offenen Thematik natürlich nahe. Die epideiktische Rede ist als drittes Gegenstück zur forensischen

33 Zur Zitierweise: Die Stellen aus Sudhaus 1892 werden durch Bandnummer, Seiten-, Kolumnenund Zeilenzahl angegeben. Sofern die Ausgabe von Longo Auricchio 1977 zitiert wird, welche diejenige von Sudhaus um PHerc. 1672 und 1674 ergänzt, wird nach Buch, Kolumne und Zeilenzahl zitiert.

34 Also wohl auch die epideiktische. 
und deliberativen aber doch auch nach inhaltlichen Kategorien gefasst. ${ }^{35}$ Wie für die forensische und deliberative Rede ist ferner auch für die epideiktische ein Redner denkbar, der ohne Unterweisung erfolgreich auftritt. Philodem legt mit seiner Begrifflichkeit den Finger darauf, dass es eine Rhetorik gibt, die lehr- und lernbar und rein formal bestimmt ist. Diese Beschreibung scheint ihm der traditionelle Begriff epideiktischer Rede nicht leisten zu können. Die ,Umbenennung“ bei Philodem ist so Folge einer klaren Umwertung. Auf den Unterrichtskontext wird auch in Philod. Rh. 2 (PHerc. 1674), col. 28,13-25 zurückgegriffen:

Tò $\delta \grave{\varepsilon} \pi \alpha \rho \alpha \tau \varepsilon-$

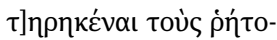

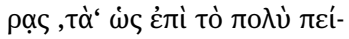

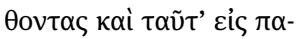

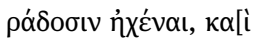

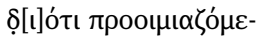

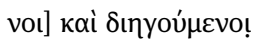

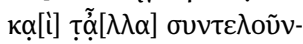

$\tau \varepsilon \varsigma \dot{\varepsilon} \xi[\tilde{\eta} \varsigma] \mu \alpha \dot{\alpha} \lambda ı \sigma \alpha \pi \varepsilon^{\prime}-$

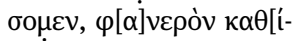

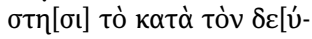

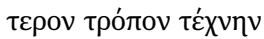

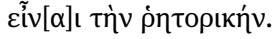

Die Beobachtung, dass die Redner meistens überzeugen, und der Ruf bezüglich ihres Vortrags, und der Umstand, dass wir überzeugen, weil wir ein Vorwort verfassen und einen Bericht und das Übrige ${ }^{36}$ der Reihe nach ausführen, all dies macht es offensichtlich, dass die Rhetorik eine Wissenschaft der zweiten Art ist.

Philodem setzt hier die gängigen Begriffe innerhalb der zweiten Redneraufgabe der

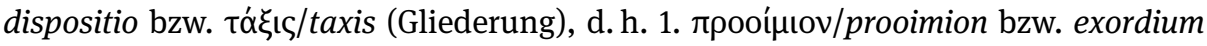

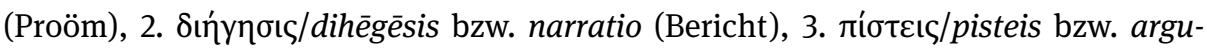

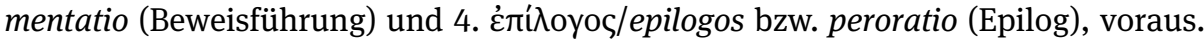
Mit dem regelhaften Aufbau einer Rede verbindet er das gängige Ziel der Rhetorik, die Überzeugung ( $\pi$ íotıৎ/pistis). Wir können folgern, dass nach Philodem der rhetorische Redeaufbau der Überzeugung wesentlich Vorschub leistet und so die Wissen-

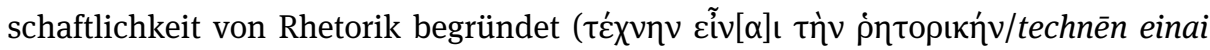

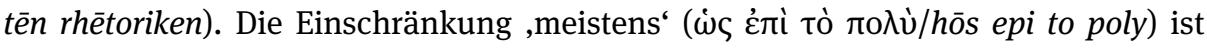
eine Folge der Bestimmung, dass auch formale Rhetorik nur eine Wissenschaft ,der

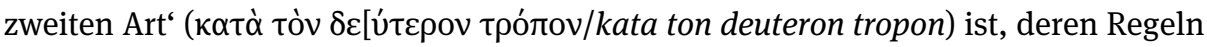

35 Zum Inhalt epideiktischer Rede äußern sich Quintilian, inst. 3,7,1-28 und Fortunatianus, rhet. 1,15 f.: Quod est demonstrativum genus? - Cum aliquid demonstramus, in quo est laus et vituperatio („Was ist die epideiktische Rede? - Wenn wir etwas zeigen, worin Lob und Tadel liegen“).

36 Also die auf den Bericht folgende Argumentation und der Redeschluss. 
keinen festen Gesetzmäßigkeiten folgen. Philodem sieht daher Rhetorik wie Medizin und Seefahrt als stochastische Wissenschaft. ${ }^{37}$

Zur Konkurrenz zwischen Rhetorik und Philosophie in der Jugenderziehung, die nach Liebersohn 2010 überhaupt als Hintergrund der Debatte um die Wissenschaftlichkeit von Rhetorik zu sehen ist, nimmt Philodem indirekt in $R h .1,174$, col. 14,1924 und col. 15,1-18 Stellung, wenn er den richtigen Metapherngebrauch als Gegenstand des Philosophieunterrichts betrachtet. Überhaupt sind der vorgeschlagenen

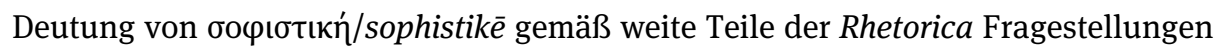
gewidmet, die wir als stilistische verstehen wollen, ${ }^{38}$ wenn auch in spezifisch epikureischer Prägung. Denn es fällt auf, dass Philodem von den Redetugenden, wie sie Theophrast zugeschrieben werden, nur die Klarheit ( $\sigma \alpha \varphi \eta ́ v \varepsilon ı /$ saphēneia), erwähnt. ${ }^{39}$ In Philod. Rh. 1,152, col. 9,1-22 sieht Milanese 1989, 87-95 gar eine Polemik gegen Handbücher zur Stilistik. Ebenso wenig wie sich Philodem an den aristotelischen Redegattungen orientiert, scheint er hier eine Rezeption von Theophrasts Stilistik zu bieten. In Rh. 1,369, col. 86,9-17 begründet Philodem ferner die Klarheit nicht stilistisch, sondern aufgrund der Durchdringung des Inhalts durch den Redner. Noch deutlicher wird diese Haltung in $R h$. 1,149, col. 4,1-14 und 151, col. 7,7-11, wo er ausgehend vom philosophischen Konzept des Vorbegriffs ( $\pi \rho{ }^{\prime} \lambda \eta \psi \mathrm{s} /$ prolèpsis) den natürlich schönen Ausdruck ${ }^{40}$ anführt, den er dem Philosophen, nicht aber dem Redner zuweist. Der Sprachgebrauch des Philosophen gilt als Ideal auch in $R h$. 1, col. 4,10-22 und Rh. 2,27, col. 32.18,3-15. Nur wenn Klarheit des Inhalts zugrunde liegt, kann auch die Darstellung und ihre Stilistik klar sein. Der epikureische Redner-Philosoph versteht es, den Inhalt stilistisch so umsetzen, dass er direkt über

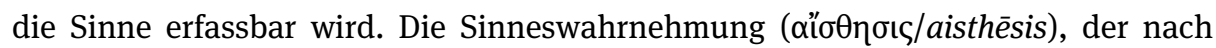
epikureischer Erkenntnislehre Wahrheitsgehalt zukommt, und Ästhetik gehen bei Philodem und auch Lukrez (man denke an das Buchstabengleichnis in Lucr. 1,822829; 1,897-920 und 2,1013-1022) einher. ${ }^{41}$ Soweit konnten wir sehen, dass für Philodem der Philosoph der beste Redner ist, weil er allein die rhetorische Tugend der Klarheit umzusetzen vermag. Daran schließt sich die Feststellung an, dass Philodem

37 Philod. Rh. 2 (PHerc. 1674), col. 2,15-24, col. 3,16, col. 6,3-8, col. 6,25-31 und col. 26,3-16. Der Vergleich von Medizin und Dichtung, auf dem auch das Katharsis-Konzept bei Aristoteles gründet (vgl. Flashar 1956), lässt sich aber auch in der weiteren Diskussion bei Philodem beobachten. Denn in De loquendi libertate wird die Ähnlichkeit affektlenkender Rede und ihrer nicht gänzlich prognostizierbaren Wirkung auf den Geist des Rezipienten zur Medizin und ihrer Wirkung auf den Körper des Patienten wiederholt in Vergleichen und Metaphern etabliert; vgl. Philod. L.L. col. 17a; fr. 63 f.; 69. Philodem nimmt also in den Rhetorica und in De loquendi libertate einen Vergleich auf, der für Dichtung bereits akzeptiert ist, und nähert beide, Rhetorik und Dichtung, in ihrer Wirkung auf den Geist an.

38 Vgl. aber Gaines 1985, der sich den Stellen widmet, an denen Philodem auch auf die inventio zu sprechen kommt.

39 Milanese 1989; vgl. Diog. L. 10,13 zu Epikur.

40 In Poem. 5, col. 30 zieht Philodem das Konzept natürlich schöner Dichtung heran.

41 Beer 2010. 
nicht anders als seine stoischen Mitstreiter die Wissenschaftlichkeit von Rhetorik nur durch ihre Verortung in der Philosophie, indem sie als Qualifikationsmerkmal des stoischen bzw. epikureischen Weisen gilt, gewinnen kann.

Sophistische Rhetorik, so konnten wir sehen, ist an den Kontext philosophischer Unterweisung gebunden und zeigt sich in der Konzentration auf reine Spracharbeit unter Vernachlässigung spezifischer Inhalte wie Politik und Forensik. Sie ist die Rhetorik, die sich auch der epikureische Philosoph zunutze machen kann. Mit der Bestimmung der Spracharbeit als Kernaufgabe der Rhetorik ergibt sich die Frage, wie Philodem die Abgrenzung zwischen Rhetorik und Dichtung abgesehen vom Metrum unternimmt. Ein Vergleich zwischen De poematis und Rhetorica macht deutlich, dass Philodem tatsächlich mehr Ähnlichkeiten als Unterschiede aufzeigt. Beide, Rhetorik (Rh. 2,289, fr. 13b,1-9) und Dichtung (Poem., tractatus tertius (PHerc. 1074+1081+1676), col. 17), lenken die Affekte des Rezipienten, indem sie Vergnügen bereiten. Explizit bestimmt Philodem ihren Bezug in Poem. 5, col. 7, wo es heißt,

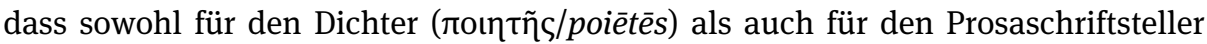

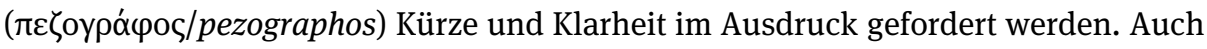
in Poem. 5, col. 12 lehnt Philodem den Versuch eines Kritikers, gute Dichtung durch ihren gleichmäßigen und mit den Figuren und dem Inhalt übereinstimmenden Stil zu definieren, mit dem Urteil ab, dass dies auch für Prosa gelte. Was ferner Demetrios von Byzanz zur Übereinstimmung zwischen Wortwahl und Thematik des Inhalts sowie zum Stil überhaupt schreibe, das gelte, so Philodem in Poem. 5, col. 13, für einen jeglichen Ausdruck. Ebenso werden in Poem. 5, col. 30-32 Anschaulichkeit, Klarheit, Kürze, Angemessenheit und Schmuck des Ausdrucks als von anderen Kritikern angeführte stilistische Tugenden von Dichtung genannt. Philodem bringt hier den für ihn beinahe schon standardmäßigen Vorbehalt, dass all dies, was so einzeln

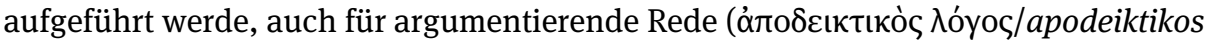
logos) gelte. ${ }^{42}$ Anders als in den Rhetorica, wo der Philosoph zum besten Redner wird, schwingt sich Philodem in De poematis allerdings nicht zum Standpunkt auf, dass der Philosoph der beste Dichter sei - zumindest nicht in den überlieferten Fragmenten.

\section{Von Rhetorik zur Kunstprosa: Der Fall $\pi \varepsilon p i$

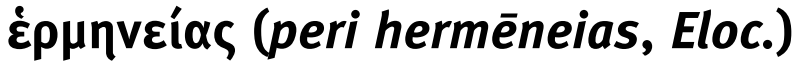

Wir können mit Fortenbaugh 2001, 225 also eine Verbindung von Philosophie und Rhetorik bei Philodem konstatieren. Wir können aber noch weiter feststellen, dass diese Überführung philosophischer Rede in das Gebiet der Rhetorik mit einer Öffnung der Rhetorik gegenüber literatur-, ja dichtungskritischen Fragestellungen ein-

42 Ähnliches folgert Chandler 2006, 143 f. für Philod. Rh. 2 (PHerc. 1672), col. 22,29-39. 
hergeht, indem die inhaltliche Bestimmung von Rhetorik zugunsten der formalen aufgegeben wird. Neben Philodem habe, so Fortenbaugh 2001, 225 gerade Demetrius von Phaleron mit De elocutione philosophische Rede als Teil der Rhetorik eta-

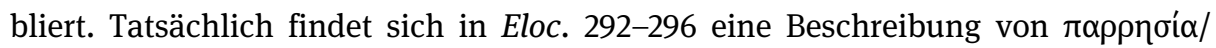
parrhēsia (die üblicherweise vom Philosophen erwartete freimütige Rede) und wird in 297 die sokratische Gesprächsführung in Abgrenzung von der Redeweise Aristipps oder Xenophons erläutert. ${ }^{43}$ Mit De elocutione liegt ferner eine Erörterung zur Stilistik von Prosa vor, deren Distanz zur oral rhetorischen Praxis (noch weit mehr als die Nähe zur Philosophie) ins Auge springt und die im Folgenden vorgestellt werden soll, weil sie das Ziel der Richtung, in die sich Theophrast und Philodem in ihrer rhetorischen Reflexion bewegen, illustriert. Die Zuordnung von De elocutione zur Rhetorik ist geläufig. ${ }^{44}$ Im Folgenden soll hingegen die Nähe zur Dichtungskritik hervorgehoben werden, um die für die hellenistische Rhetorik-Theorie charakteristische Verbindung zu verdeutlichen.

Die Zuschreibung zum Theophrast-Schüler Demetrios von Phaleron ist in der Forschung nicht abschließend diskutiert. Die überwiegende Mehrheit, darunter Wehrli 1968, Rhys Roberts 1969 und Chiron 2001, spricht sich gegen Demetrios von Phaleron als Autor aus, während Fortenbaugh 2001 ihn als Autor behandelt. Die Assoziation zum Theophrast-Schüler wird begründet 1. durch die Konzentration auf Stilistik, 2. durch den Umstand, dass Diogenes Laertios zu Demetrios von Phaleron

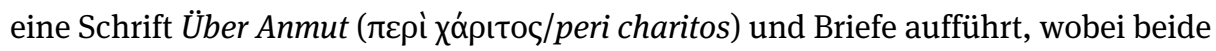
Themen in De elocutione behandelt werden, und 3. aufgrund einer Anekdote zu Demetrios von Phaleron im Text selbst, welche ein Beispiel für den im Kontext beschriebenen heftigen Stil bietet. Bezüglich der Anekdote scheint es aber nur natürlich, nicht davon auszugehen, dass Demetrios von Phaleron selbst der Autor ist. Chiron 2001, 32 fasst die Datierungsüberlegungen zum Paradox zusammen, dass die Sprache spät, der Inhalt aber alt sei, und kommt auf eine Datierung zwischen Demetrios von Phaleron und Cicero, und damit, so können wir folgern, in die Zeit alexandrinischer Dichtungskritik. Michel 1982 hat, neben alexandrinischen Zügen in De elocutione, Einflüsse auf Theokrit festgestellt. ${ }^{45}$ Anders favorisiert Calcante $2000^{46}$ aufgrund ihres Vergleichs von De elocutione mit den Traktaten von Dionysios von Halikarnassos und Pseudo-Longinos das 1. Jh. n. Chr. Diogenes Laertios (5,83 f.)

\footnotetext{
43 Ein Blick auf Gell. 18,4 zeigt, dass die sokratische Gesprächsführung im 2. Jh. n. Chr. auch zu den Mitteln eines Redelehrers wie Sulpicius Apollinaris gehörte.

44 Vgl. Chiron 2001, 41 und Fortenbaugh 2001, 225.

45 So Michel 1982, 51. Die alexandrinischen Züge sind zu ergänzen durch Dem. Eloc. 170, wo ein Lob auf die Linse ( wird. Der Titel dieser nicht überlieferten Rede lässt an die alexandrinische Favorisierung der Kleinform und zugleich an die Vermeidung breitgetretener Themen denken (Callim. fr. 1,1 bzw. 1,27f. Pfeiffer) und steht in Eloc. 170 zur Illustration kynischer Satire, die als Parodie wie die alexandrinische Kleinform die Gegenperspektive auf einen bekannten Gegenstand eröffnet.

$46 \mathrm{Ihm}$ folgt Marini 2007.
} 
nennt zwanzig weitere Persönlichkeiten mit Namen Demetrios, von denen insbeson-

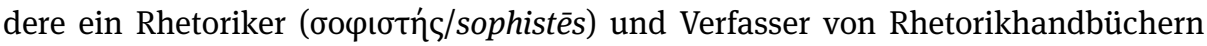
aus Alexandria als Autor von De elocutione in Betracht gezogen werden sollte. Im-

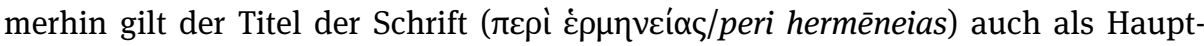

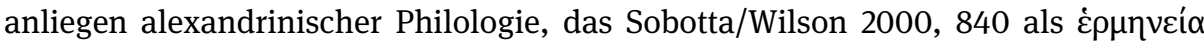

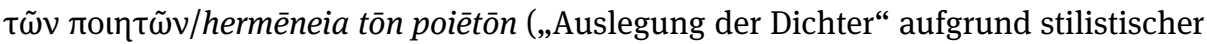
Beobachtungen) bestimmen. Zuordnungsversuche sollten ferner Philodem (Poem. 5, col. 13) berücksichtigen, der unter den zahlreichen Kritikern, gegen die er sich wendet, auch (wie Diog. L. 5,83) einen Demetrios von Byzanz nennt. Dieser habe für gute Dichtung die, wohl stilistische, Übereinstimmung von Wortwahl und Inhalt gefordert. Demetrios von Byzanz hat damit gemäß Philodem eine Vorstellung geäußert, die wir im Folgenden als zentralen Bestandteil der in De elocutione vertretenen Stilkritik bestimmen können.

$\mathrm{Zu}$ den augenfälligsten Neuerungen in De elocutione gehört die Unterteilung von Rede in vier Stile, die als erhaben ( $\mu \varepsilon \gamma \alpha \lambda$ олрєли́ $/$ megaloprepēs), elegant ( $\gamma \lambda \alpha-$

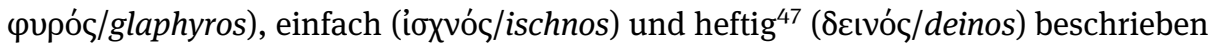
werden. ${ }^{48}$ Sie schlagen sich nieder in den drei Ebenen der Wortwahl ( $\lambda \varepsilon \dot{\xi} \xi \varsigma /$ lexis),

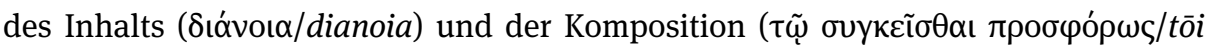
synkeisthai prosphorōs). ${ }^{49}$ Im Anschluss an den einfachen Stil wird der dem Brief angemessene Stil abgehandelt, der sich durch eine etwas feinere Ausformulierung, als sie im Dialog üblich ist, auszeichnet. ${ }^{50}$ Jedem Stil wird abschließend seine Pervertierung gegenübergestellt.

Die angesprochene Distanz zur praktischen Rhetorik wird zum einen in der Vierteilung offenbar, die von der Dreiteilung der aristotelischen Redegattungen abweicht. Zum anderen zeugt der Brief als schriftliches Genre von einem unkonventionellen Zugang zu Rhetorik. Ferner geht De elocutione zwar von einem Hörer

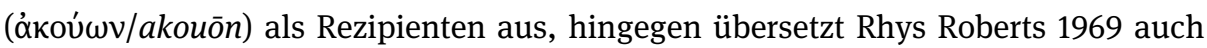
(so in Eloc. 45 und 216) mit ,Leser', werden doch als stilistische Vorbilder neben den Rednern Polykrates, Lysias, Antisthenes, Isokrates, Aischines, Demosthenes, Demades und dem Historiker Theopomp auch die Schriftsteller und Philosophen Heraklit, Herodot, Platon, Thukydides, Xenophon, Aristoteles, Ktesias (der Arzt und

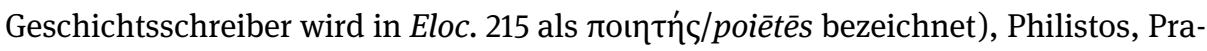
xiphanes, Dikaiarchos, Kleitarchos, Krates und Artemon und sogar die Dichter Homer, Sappho, Theognis, Hipponax, Sophron, Aristophanes, Menander, Philemon und Sotades genannt. Die Beobachtung, dass sich De elocutione hin zur Formulie-

47 „Heftig“ entspricht der gängigen Übersetzung, wobei unter Berücksichtigung des Bedeutungs-

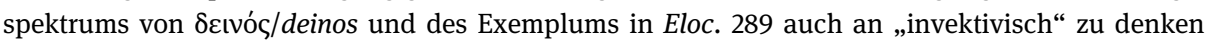
wäre.

48 Eloc. 36.

49 Eloc. 38.

50 Eloc. 224. 
rung einer Kunstprosa bewegt, wird außerdem gestützt durch die Terminologie zu

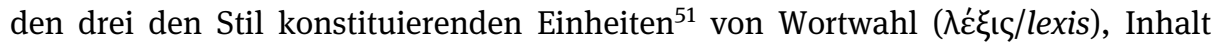

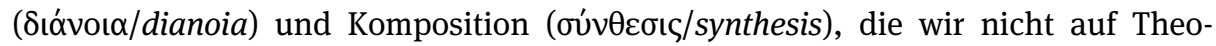
phrast zurückführen können. Terminologie und Konzeption stimmen vielmehr mit Philodems Hauptanliegen in De poematis überein, wonach sich gute Dichtung dadurch auszeichnet, dass die Komposition, d. h. die Formulierung, im Dienste einer

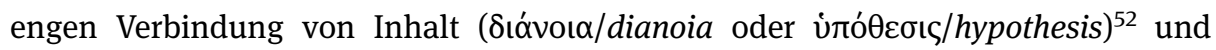

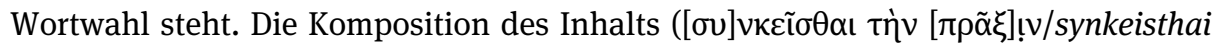

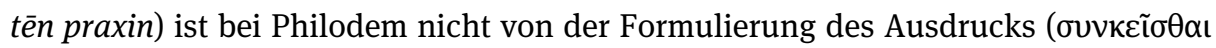

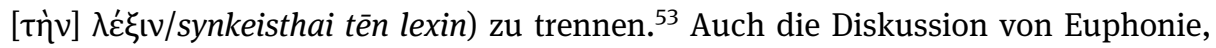
die in Eloc. 175 attische Schriftsteller auszeichnet, ebd. 69 als Ziel jeder Rede genannt und wohl auch ebd. 199 hinter dem gängigen Verfahren der Umformulierung eines Ausdrucks zur Bestimmung seines Wohlklangs zu denken ist, teilt De elocutione mit Philodems De poematis. Ferner werden in De elocutione wiederholt Dichtung und Rhetorik verbunden, etwa wenn ebd. 286 das poetische Moment in den Reden von Demades hervorgehoben wird, und ebd. 112 die Meinung geäußert wird, dass ein poetischer Ausdruck den Prosa-Stil erhöhe. Auch die Vergleiche mit dem Bildhauer Pheidias in Eloc. 14 bzw. dem Maler Nikias ebd. 76 machen die Anlehnung an den Diskurs der Dichtungskritik offenbar. ${ }^{54}$ Als letztes Argument dafür, dass in De elocutione weniger traditionelle Rhetorik, sondern eher der Stil von Kunstprosa verhandelt wird, soll hier schließlich die Beobachtung stehen, dass die Schrift als Verbindung von Rhetorik und Poetik in einem Kodex zusammen mit Aristoteles' Rhetorik und Poetik (sowie Dionysios von Halikarnassos, De Compositione und Ad Ammaeum) überliefert wurde. ${ }^{55}$

Als Movens rhetorischer Reflexion im Hellenismus konnten wir eingangs die Debatte zwischen Philosophie und Rhetorik bestimmen, in der sich Rhetorik als wissenschaftliches Fach einem Rechtfertigungsdruck ausgesetzt sah. Die Debatte kann auf die Konkurrenzsituation zwischen Rhetorik und Philosophie in der Jugenderziehung zurückgeführt werden. Durch die Betonung der Stilistik stellt sich Rhetorik in eine Linie mit den in ihrer Bedeutung in der Antike eminenten Unterrichtsfächern Sprache und Literatur. Die erfolgreiche Behauptung von Rhetorik als Studienfach und die im Zuge dieser Bemühungen zu sehende Entwicklung einer Kunstprosa können mit als Grund gelten für die Rhetorisierung antiker Literatur, insbesondere des Romans.

51 Eloc. 10; vgl. zur Beschreibung von Inhalt, Wortwahl und Komposition in De elocutione auch Chiron 2001, 39.

52 Der Begriff steht bei Philodem in anderer Bedeutung als bei Hermagoras.

53 So etwa Philod. Poem. 5, col. 15 und Poem., tractatus tertius (PHercc. 1074+1081+1676), col. 1719). Daher hält Philodem auch nichts von der Metathese als Praxis der Literaturkritik.

54 Dazu Männlein-Robert 2007, 2, die bildende Kunst wenn nicht als Schwesterkunst, so doch als Projektionsfläche für Dichtung und Dichtungstheorie besonders im Hellenismus beschreibt.

55 Rhys Roberts 1969, 209. 


\section{Literatur}

Arrighetti, Graziano (2000): Filodemo fra poesia, mito e storia. In: Michael Erler (Hg.):

Epikureismus in der späten Republik und der Kaiserzeit. Stuttgart, 13-31.

Arrighetti, Graziano (2016): Filodemo, le technai e la retorica. In: Irmgard Männlein-Robert u. a.

(Hg.): Philosophus Orator. Rhetorische Strategien und Strukturen in philosophischer

Literatur. Basel, 181-201.

Barwick, Karl (1963): Das rednerische Bildungsideal Ciceros. Berlin.

Beer, Beate (2010): Lukrez und Philodem. Poetische Argumentation und poetologischer Diskurs. Basel.

Calcante, Cesare Marco (2000): Genera dicendi e retorica del Sublime. Pisa/Rom.

Chandler, Clive (Hg.) (2006): Philodemus. On rhetoric. Books 1 and 2. New York/London.

Chiron, Pierre (2001): Un rhéteur méconnu: Démétrios (Ps.-Démétrios de Phalère). Essai sur les mutations de la théorie du style à l'époque hellénistique. Paris.

Erler, Michael (1992): Orthodoxie und Anpassung. Philodem, ein Panaitios des Kepos? In: Museum Helveticum 49, 171-200.

Erler, Michael (2007): Platon. In: Hellmut Flashar (Hg.): Grundriss der Geschichte der Philosophie. Begründet von Friedrich Ueberweg. Völlig neu bearbeitete Ausgabe. Die Philosophie der Antike. Bd. 2/2. Basel.

Ferrario, Matilde (1981): La concezione della retorica da Epicuro a Filodemo. In: Proceedings of the sixteenth international Congress of Papyrology. Chicago, 145-152.

Flashar, Hellmut (1956): Die medizinischen Grundlagen der Lehre von der Wirkung der Dichtung in der griechischen Poetik. In: Hermes 84, 12-48.

Fortenbaugh, William W. (1985): Theophrastus on Delivery. In: William W. Fortenbaugh (Hg.): Theophrastus of Eresus. On his Life and Work. Bd. 2. Brunswick/New York, 269-288.

Fortenbaugh, William W. (Hg.) (1992): Theophrastus of Eresus. Sources for his life, writings, thought and influence. Vol. 2. Leiden/New York/Köln.

Fortenbaugh, William W. (1994): Theophrastus, the Characters and Rhetoric. In: William W. Fortenbaugh/David C. Mirhady (Hg.): Peripatetic Rhetoric after Aristotle. New Brunswick/New York, 15-35.

Fortenbaugh, William W./Eckart Schütrumpf (Hg.) (2001): Demetrius of Phalerum. Text, Translation and Discussion. New Brunswick/London.

Fortenbaugh, William W. (2003): Theophrastus on Delivery. In: William W. Fortenbaugh (Hg.): Theophrastean Studies. Stuttgart, 253-271.

Fortenbaugh, William W. (2005): Cicero as a Reporter of Aristotelian and Theophrastean Rhetorical Doctrine. In: Rhetorica 23, 37-64.

Fuhrmann, Manfred (1995): Die antike Rhetorik. Eine Einführung. 4. Aufl. München.

Gaines, Robert N. (1985): Philodemus on three activities of rhetorical invention. In: Rhetorica 3/3, $155-163$.

Grube, George Maximilian Antoine (1952): Theophrastus as a Literary Critic. In: Transactions of the American Philological Association 83, 172-183.

Innes, Doreen C. (1985): Theophrastus and the Theory of Style. In: William W. Fortenbaugh (Hg.): Theophrastus of Eresus. On his Life and Work. Bd. 2. Brunswick/New York, 251-267.

Innes, Doreen C. (1994): Period and Colon: Theory and Example in Demetrius and Longinus. In: William W. Fortenbaugh/David C. Mirhady (Hg.): Peripatetic Rhetoric after Aristotle. New Brunswick/New York, 36-53.

Kasulke, Christoph Tobias (2005): Fronto, Marc Aurel und kein Konflikt zwischen Rhetorik und Philosophie im 2. Jh. n. Chr. Berlin.

Kennedy, George A. (1994): A new history of classical rhetoric. Princeton.

Liebersohn, Yosef Z. (2010): The Dispute concerning Rhetoric in Hellenistic Thought. Göttingen (Hypomnemata 185). 


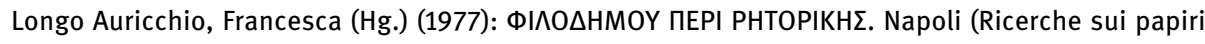
ercolanesi, 3).

Mal-Maeder, Danielle van (2007): La fiction des déclamations. Leiden.

Männlein-Robert, Irmgard (2007): Stimme, Schrift und Bild. Zum Verhältnis der Künste in der hellenistischen Dichtung. Heidelberg.

Mangoni, Cecilia (Hg.) (1993): Philodemus: Il quinto libro della Poetica (PHerc. 1425 e 1538). Napoli.

Marini, Nicoletta (Hg.) (2007): Demetrio. Lo stile. Rom.

Michel, Alain (1982): La parole et la beauté: rhétorique et esthétique dans la tradition occidentale. Paris.

Milanese, Guido (1989): Lucida carmina. Comunicazione e scrittura da Epicuro a Lucrezio. Mailand.

Roberts, William Rhys (Hg.) (1902): Demetrius. On style. Cambridge. [Nachdruck Hildesheim 1969.]

Sobotta, Raphael/Nigel Wilson (2000): Philologie. In: Der Neue Pauly 9, 836-844.

Sudhaus, Siegfried (Hg.) (1892): Philodemi Volumina Rhetorica. Leipzig.

Wehrli, Fritz (Hg.) (1968): Die Schule des Aristoteles. Texte und Kommentar. 2. Auflage. Basel.

Woerther, Frédérique (Hg.) (2012): Hermagoras. Fragments et témoignages. Paris. 
Bereitgestellt von | UZH Hauptbibliothek / Zentralbibliothek Zürich 